\title{
Changing treatment paradigms for the manage- ment of inflammatory bowel disease
}

Jong Pil Im ${ }^{1}$, Byong Duk Ye², You Sun $\mathrm{Kim}^{3}$, and Joo Sung Kim

\begin{abstract}
${ }^{1}$ Department of Internal Medicine and Liver Research Institute, Seoul National University College of Medicine, Seoul; ${ }^{2}$ Department of Gastroenterology, Asan Medical Center, University of Ulsan College of Medicine, Seoul; ${ }^{3}$ Department of Internal Medicine, Inje University Seoul Paik Hospital, Seoul, Korea
\end{abstract}

Received: December 4, 2017

Accepted: December 12, 2017

\section{Correspondence to}

Joo Sung Kim, M.D.

Department of Internal Medicine and Liver Research Institute, Seoul National University College of Medicine, 101 Daehak-ro, Jongno-gu, Seoul 03080, Korea

Tel: +82-2-740-8112

Fax: +82-2-742-8601

E-mail:jooskim@snu.ac.kr

This paper was contributed by Korean Association for the Study of Intestinal Diseases.
Inflammatory bowel disease (IBD) is a chronic and progressive inflammatory condition of the gastrointestinal tract causing bowel damage, hospitalizations, surgeries, and disability. Although there has been much progress in the management of IBD with established and evolving therapies, most current approaches have failed to change the natural course. Therefore, the treatment approach and follow-up of patients with IBD have undergone a significant change. Usage of immunosuppressants and/or biologics early during the course of the disease, known as top-down or accelerated step-up approach, was shown to be superior to conventional management in patients who had been recently diagnosed with IBD. This approach can be applied to selected groups based on prognostic factors to control disease activity and prevent progressive disease. Therapeutic targets have been shifted from clinical remission mainly based on symptoms to objective parameters such as endoscopic healing due to the discrepancies observed between symptoms, objectively evaluated inflammatory activity, and intestinal damage. The concept of treat-to-target in IBD has been supported by population-based cohort studies, post hoc analysis of clinical trials, and meta-analysis, but more evidence is needed to support this concept to be applied to the clinical practice. In addition, individualized approach with tight monitoring of non-invasive biomarker such as C-reactive protein and fecal calprotectin and drug concentration has shown to improve clinical and endoscopic outcomes. An appropriate de-escalation strategy is considered based on patient demographics, disease features, current disease status, and patients' preferences.

Keywords: Inflammatory bowel diseases; Prognosis; Treat-to-target; Therapeutic drug monitor

\section{INTRODUCTION}

Inflammatory bowel disease (IBD) including ulcerative colitis (UC) and Crohn's disease (CD) is a chronic and progressive inflammatory condition of the gastrointestinal tract. IBD is known to be associated with a genetic predisposition to the disease, environmental exposure, and the subsequent dysregulated immune responses [1]. The number of people diagnosed with IBD continues to increase and is the highest in Western countries; however, a significantly increased incidence is being observed in the Asian population secondary to rapid urbanization $[2,3]$.

Although there has been much progress in the management of IBD with established and evolving therapies including the use of monoclonal antibodies, most current approaches that are directed only toward symptom control have failed to change the natural course of the 
Table 1. Concept change of inflammatory bowel disease

\begin{tabular}{ll}
\hline Old concept & \multicolumn{1}{c}{ New concept } \\
\hline IBD is a disease with intermittent flare & IBD is progressive in a substantial proportion of patients \\
Treat all patients in the same way & Individualized approach according to severity and prognosis \\
Treat to targeting clinical remission and response & Treat to targeting more objective outcomes, e.g., mucosal healing \\
Reactive management with intermittent monitoring & Proactive management with tight monitoring \\
\hline
\end{tabular}

IBD, inflammatory bowel disease.

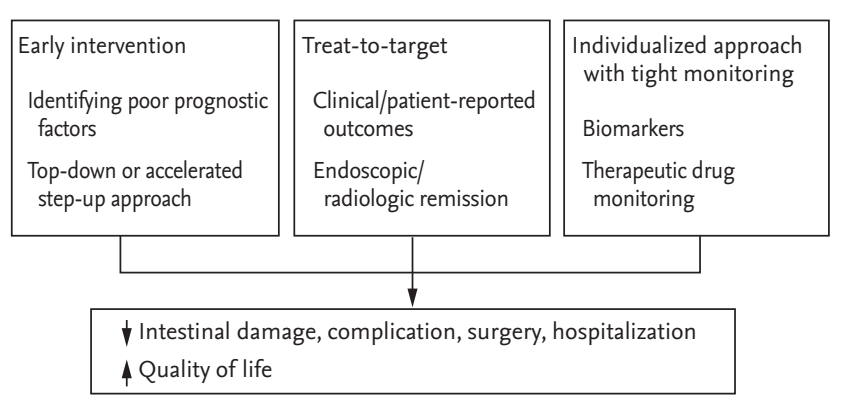

Figure 1. Current strategies for the management of inflammatory bowel disease.

disease $[4,5]$. A population-based study has shown that the probability of CD-related surgery was as high as 96.7\% in anti-Saccharomyces cerevisiae antibody-positive patients aged $<20$ years who had been diagnosed with penetrating disease and had been initially treated with systemic steroids [6]. Additionally, disability caused by IBD correlates with disease severity, and it has been observed that improved medication compliance is associated with lower disability and a better quality of life [7]. The heterogeneity in clinical features and prognosis makes it difficult to use generally effective strategies for the treatment of this condition, and consequently contributes to a wide variation in strategies used for IBD management [8].

Recently, the treatment approach and follow-up of patients with IBD have undergone a significant change (Table 1). It is now widely acknowledged that IBD is a progressive disease causing intestinal damage and disability, which therefore mandates the institution of prompt individualized treatment during the early stages to prevent irreversible complications and produce better outcomes with reduced rates of hospitalization and surgery [9]. This review encompasses the emerging trends in the treatment paradigm of IBD including prognostication and early intervention, treating to target, tight monitoring, and adoption of individualized therapeutic approaches (Fig. 1).

\section{EARLY INTERVENTION BASED ON PROGNOS- TIC FACTORS}

Recently, top-down or accelerated step-up treatment strategies have been introduced to modify the natural course of IBD aimed at achieving better outcomes. There exists a window of opportunity in the early stages of IBD, particularly in CD patients to reduce bowel damage, hospitalizations, surgeries, and disability as has been observed in patients diagnosed with rheumatoid arthritis [9]. Administration of immunosuppressants and/or biologics early during the course of the disease was shown to be superior to conventional management in patients who had been recently diagnosed with IBD in terms of better mucosal healing $(\mathrm{MH})$, induction of steroid-free remission, and prevention of hospitalization [10-12].

A hospital-based CD cohort study in Korea showed an intestinal resection rate that matched the rate observed in Western countries, and the surgical rate was observed to decrease significantly over 30 years following the early usage of thiopurines [13]. This trend of a decreased colectomy rate in Korean UC patients was associated with more frequent and earlier use of thiopurines and anti-tumor necrosis factor (TNF) agents over time [14].

Despite these benefits, it is unclear whether such aggressive treatment is better than conventional step-up therapy for the prevention of adverse outcomes. Additionally, a significant percentage of patients showed clinical remission over 5 years and remained free from complications after 10 years of diagnosis even during the pre-biologic era [15]. Moreover, drug-related adverse events, high cost, and issues related to generalizability 
and applicability to clinical practice are concerns associated with the adoption of the top-down or accelerated step-up approach in routine clinical practice [16].

The Randomised Evaluation of an Algorithm for Crohn's Treatment (REACT) study was an open-label cluster-randomized controlled trial that compared the efficacy and safety of early combined immunosuppression (ECI) with the use of anti-TNF agents and antimetabolites versus conventional management of $\mathrm{CD}$ among gastroenterology practices in the community. Although ECI did not show greater benefit in terms of clinical remission than that observed with conventional management, ECI reduced major adverse events such as the need for surgery, hospital admissions, or serious CD-associated complications. ECI did not increase the risk of serious treatment-related adverse events or mortality. Despite the limitations of an un-blinded study design and assessment of outcome measures based on evaluation of subjective symptoms, these results suggest that highly effective therapy initiated early in the course of the disease might change the natural course of $\mathrm{CD}$ without a significant increase in drug-related risks [16].

The effects of early administration of anti-TNF/immunomodulator (IM) therapy were retrospectively evaluated in Korean CD patients presenting with $\geq 2$ poor prognostic factors. Poor prognostic factors were: age < 40 years at the time of diagnosis, early use of systemic steroid, and the presence of a perianal fistula at the time of diagnosis. The early treatment group was defined as patients who were administered anti-TNF and/or IM treatment within 2 years of $\mathrm{CD}$ diagnosis. The early treatment group presenting with poor prognostic factors demonstrated better outcomes, which were indicated by a greater time interval until the need for intestinal surgery and fewer complications than those observed in the late treatment group [17].

Prognostic predictors of colectomy in patients presenting with UC are: extensive disease, proximal extension of lesions during the disease course, extra-intestinal manifestations, younger age at the time of disease onset, severity of inflammation, and a poor response to treatment [5,18]. Poor prognostic factors associated with CD include: younger age, presence of strictures and/or penetrating lesions, jejunal or perianal involvement at the time of diagnosis, systemic steroid use, and/or smoking habits/history. The recently developed Korean CD prediction model based on clinical parameters assessed at the time of diagnosis, serves as an accurate predictor of a patient's probability of undergoing CD-related surgery [19]. This kind of predictive model based on an assessment of poor clinical factors can be used to stratify patients into subgroups and identify those who would benefit from early individualized intervention among newly diagnosed patients to control disease activity and prevent progressive disease.

\section{TREAT-TO-TARGET}

Therapeutic targets have been defined for chronic diseases such as diabetes, hypertension, and rheumatoid arthritis. With respect to IBD, the concept of treat-totarget originated from the discrepancies observed between symptoms, objectively evaluated inflammatory activity, and intestinal damage, which is more commonly observed in those presenting with CD than with UC. Various studies have shown that a full Mayo score including the objective endoscopic grade shows a relatively high correlation with disease severity of UC. However, a poor correlation was frequently observed between the endoscopic disease activity and the symptom-based Crohn's disease activity index (CDAI) [20].

Shifting the therapeutic target from clinical remission to objective parameters such as endoscopic healing has been supported by population-based cohort studies, post hoc analysis of clinical trials, and meta-analysis [11,21-23]. The Active Ulcerative Colitis Trial (ACT)-1 and ACT2 have shown that $\mathrm{MH}$ observed in patients following 8-week treatment with infliximab demonstrated a positive correlation with better clinical outcomes including lower colectomy rates [11]. A meta-analysis involving > 2,000 patients presenting with active UC has shown that patients achieving $\mathrm{MH}$ at the time of the first endoscopic evaluation demonstrate long-term clinical remission, avoidance of colectomy, and corticosteroid-free clinical remission [22]. In patients with CD treated with adalimumab, deep remission at 12 weeks, a composite of clinical and endoscopic remission was significantly associated with fewer dose adjustments, hospitalizations, and CD-related surgeries, as well as an improved quality of life and physical function at 1 year [23].

Recently, the Selecting Therapeutic Targets in Inflam- 
matory Bowel Disease (STRIDE) program was initiated to determine the goals for treat-to-target strategies in patients diagnosed with IBD in routine clinical practice. Based on evidence and expert consensus, 12 goals have been developed as treat-to-target strategies. The selected targets for both UC and CD are clinical/patient-reported outcomes and endoscopic remission. In patients diagnosed with $\mathrm{CD}$, resolution of inflammation identified by cross-sectional imaging is a target, when endoscopic assessment cannot be performed. Histopathological remission has been considered an adjunctive target for UC, and biomarker remission (a C-reactive protein [CRP] and fecal calprotectin [FC] within the reference range) has been considered an adjunctive target for $\mathrm{CD}$. International consensus suggests both composite end points including clinical and endoscopic remission and the frequency of assessment to reach the target [24].

However, the applicability of the treat-to-target approach may be difficult in clinical practice. Most Asian physicians use the partial Mayo score or the Truelove and Witts severity index, which primarily relies upon symptom category to assess the disease activity of UC at the time of diagnosis and during subsequent follow-up. Regarding CD, the CDAI is used by most Asian physicians as the gold standard to determine disease activity; however, recent surveys have shown wide variations among physicians in adopting endoscopic and/or radiological evaluations as objective parameters that accurately reflect inflammatory activity [25]. Additionally, more conclusive evidence is needed to support this concept, and clinicians should remain mindful of a possible target change occurring in the future and the possibility of overtreatment of low-risk patients $[9,26]$.

\section{INDIVIDUALIZED APPROACH WITH TIGHT MONITORING}

Objective measures to assess inflammatory activity are important to adopt the treat-to-target strategy utilized for IBD management. The STRIDE program recommends endoscopic healing combined with clinical/patient-reported outcomes as therapeutic targets; however, biomarkers such as CRP or FC are used as an adjunctive target [24]. However, endoscopic assessment is usually performed prior to initiating or modifying treatment in patients showing a relapse or showing the development of new symptoms, or those in whom surgery is needed [27]. Moreover, the cost factor and patient compliance are primary limitations associated with regular endoscopic evaluation of $\mathrm{MH}$ due to the invasiveness associated with the procedure. Estimation of CRP and FC are well-studied noninvasive biomarkers that are widely used [28].

The Efficacy and Safety of Two Treatment Models in Subjects With Moderate to Severe Crohn's Disease (CALM) study compared the efficacy and safety of a tight control algorithm based on biomarkers such as CRP and FC in addition to clinical symptoms in patients who were naive to IM and/or biologic therapy [29]. A total of 244 patients with CD showing < 6 years of duration were randomly assigned to either a tight control or a clinical management group. The tight control group was monitored with estimation of CRP and FC in addition to clinical symptoms based on the CDAI. After 8 weeks of prednisolone therapy, their treatment regimen was escalated in a stepwise manner including the addition of adalimumab and azathioprine in both groups who met the predefined failure criteria. The primary endpoint defined as $\mathrm{MH}$ at 48 weeks was achieved in $46 \%$ of the tight control group, which was significantly higher than $30 \%$ observed in the clinical management group. This study showed that tight monitoring of objective markers, as well as clinical symptoms helped in effective decision making for the institution of appropriate treatment and therefore achieved superior clinical and endoscopic outcomes compared to the symptom-based approach.

The introduction of IMs and biologic agents has reduced the risk of surgery over time $[13,14]$. However, a significant number of patients with IBD, particularly those diagnosed with $\mathrm{CD}$ are required to undergo repeat surgery due to relapse, leading to significant morbidity and disability [30]. Thiopurine or anti-TNF therapy is considered in addition to smoking cessation and 5-aminosalicylic acid maintenance therapy to prevent postoperative relapse in patients diagnosed with $\mathrm{CD}$ [31]. The Post-Operative Crohn's Endoscopic Recurrence (POCER) study showed that individualized management based on the results of 6-month colonoscopic examination combined with an assessment of the clinical risk of recurrence was more effective than standard drug therapy in reducing postoperative recurrence [32]. Estimation 
of FC at 6,12 , and 18 months after intestinal resection was a good predictor of relapse, and monitoring of FC is a noninvasive and inexpensive technique, which could serve as a useful component of the individualized postoperative management algorithm [33].

Despite the proven efficacy of anti-TNF agents in inducing and maintaining remission, approximately $10 \%$ to $20 \%$ of IBD patients are refractory to induction therapy, and annually $13 \%$ to $24 \%$ patients report a loss of response (LOR) during maintenance therapy [34]. Therapeutic drug monitoring (TDM) combined with anti-drug antibodies (ADAs) is an important component of the individualized management strategy used for IBD patients treated with anti-TNF agents. TDM can be performed periodically in patients showing remission, as well as in those showing a primary nonresponse, secondary LOR, or drug-related adverse events to guide decision-making regarding treatment optimization [35]. Recently, a proactive approach, targeting a trough level of 5 to $10 \mu \mathrm{g} / \mathrm{mL}$ by measuring the infliximab concentration and ADA, in asymptomatic IBD patients showed better clinical outcomes including lesser need for IBD-related surgery and hospitalization than the benefits noted with reactive monitoring in patients showing symptom aggravation or suspected LOR. Proactive monitoring was also observed to be associated with a reduced risk of ADA development or infusion-related adverse events [36]. Improved clinical outcomes were also observed with the use of adalimumab, fully human anti-TNF agents, and vedolizumab, a humanized monoclonal antibody against the $\alpha 4 \beta 7$ integrin in patients in whom doses had been optimized based on the results of pharmacokinetic studies [37-39].

\section{DE-ESCALATION STRATEGY}

Healthcare costs associated with IBD management have shown a significant rise. This is because a greater number of patients are being administered biologic agents alone or concomitantly with IMs earlier in the course of the disease owing to newly emerging paradigms for the management of IBD [40]. Moreover, the risk of tuberculosis (TB), both, de novo infection and reactivation of latent TB, is higher in patients receiving anti-TNF agents, particularly in areas such as Korea, which show a high prevalence of the disease [41]. Use of thiopurines is associated with significant toxicity such as bone marrow suppression, liver toxicity, opportunistic infections, and malignancies including lymphoma and non-melanoma skin cancers [42]. Concerns about cost and toxicities have led physicians and patients to consider safe de-escalation strategies once remission has been achieved.

Several long-term trials have shown that discontinuation of therapy is associated with high relapse rates, suggesting that complete cessation of treatment can be considered and implemented only in selected patients [43]. The infliximab diSconTinuation in CrOhn's disease patients in stable Remission on combined therapy with Immunosuppressors (STORI) trial prospectively examined the risks and predictors of relapse after withdrawal of infliximab in patients receiving combined maintenance therapy with antimetabolites [44]. The relapse rate within a 1 year was approximately 50\%; however, patients having $\leq 2$ risk factors, including male gender, elevated leukocytes, an elevated CRP level, elevated FC level, and decreased hemoglobin showed only a $15 \%$ risk of relapse. Several studies have identified complicated or perianal $\mathrm{CD}$, extensive disease, clinical symptoms, elevated biomarkers, endoscopically documented severe lesions, and monotherapy as predictors of relapse following cessation of therapy [45]. However, there is no consensus regarding the optimal time and methodology with respect to cessation of this potent disease-modifying therapy. This decision needs to be individualized based on patient demographics, disease features, current disease status, and patients' preferences $[9,45]$.

\section{CONCLUSIONS}

IBD is a disease entity known to cause progressive intestinal damage and disability; thus, early intervention can alter the natural course of the disease and prevent irreversible complications. However, heterogeneity in clinical features and prognosis often prevents the use of early and effective strategies. Recent studies have determined a number of long-term prognostic factors and developed predictive models, which can identify patients who can maximally benefit from early intervention. In patients diagnosed with IBD, clinical symptoms are considered to reflect the tip of the iceberg, and symptom-driven 
management often fails to improve long-term outcomes. Lately, therapeutic targets have been shifted from clinical remission to objective parameters such as evidence of endoscopic healing. An individualized treatment approach based on close and timely monitoring using non-invasive tests combined with endoscopic and radiological assessment is essential to ensure better outcomes. The management of IBD patients has greatly improved over the last decade following the introduction of newer classes of drugs, particularly biologic agents. However, earlier administration and wider use of these drugs can lead to significant healthcare costs nationwide, as well as a higher incidence of untoward treatment-related adverse events. Therefore, the use of biologic agents should be optimized with tight monitor, and an appropriate de-escalation strategy is warranted in selected patients. Further studies are needed to validate the efficacy of these newly emerging treatment paradigms to confirm their role in improving long-term prognosis by reducing disability, need for surgery and hospitalization to improve the patient's quality of life.

\section{Conflict of interest}

No potential conflict of interest relevant to this article was reported.

\section{Acknowledgments}

This work was supported by the Research Program funded by the Korea Centers for Disease Control and Prevention (2016-E63001-02).

\section{REFERENCES}

1. Raffals LE, Chang EB. Navigating the microbial basis of inflammatory bowel diseases: seeing the light at the end of the tunnel. Gut Liver 2016;10:502-508.

2. Kaplan GG, Ng SC. Understanding and preventing the global increase of inflammatory bowel disease. Gastroenterology 2017;152:313-321.

3. Ng WK, Wong SH, Ng SC. Changing epidemiological trends of inflammatory bowel disease in Asia. Intest Res 2016;14:111-119.

4. Peyrin-Biroulet L, Loftus EV Jr, Colombel JF, Sandborn WJ. Long-term complications, extraintestinal manifestations, and mortality in adult Crohn's disease in popula- tion-based cohorts. Inflamm Bowel Dis 2011;17:471-478.

5. Torres J, Billioud V, Sachar DB, Peyrin-Biroulet L, Colombel JF. Ulcerative colitis as a progressive disease: the forgotten evidence. Inflamm Bowel Dis 2012;18:1356-1363.

6. Solberg IC, Cvancarova M, Vatn MH, Moum B; IBSEN Study Group. Risk matrix for prediction of advanced disease in a population-based study of patients with Crohn's Disease (the IBSEN Study). Inflamm Bowel Dis 2014;20:60-68.

7. Yoon JY, Shin JE, Park SH, Park DI, Cha JM. Disability due to inflammatory bowel disease is correlated with drug compliance, disease activity, and quality of life. Gut Liver 2017;11:370-376.

8. Song HK, Lee KM, Jung SA, et al. Quality of care in inflammatory bowel disease in Asia: the results of a multinational web-based survey in the 2(nd) Asian Organization of Crohn's and Colitis (AOCC) meeting in Seoul. Intest Res 2016;14:240-247.

9. Colombel JF, Narula N, Peyrin-Biroulet L. Management strategies to improve outcomes of patients with inflammatory bowel diseases. Gastroenterology 2017;152:351-361.

10. D'Haens G, Baert F, van Assche G, et al. Early combined immunosuppression or conventional management in patients with newly diagnosed Crohn's disease: an open randomized trial. Lancet 2008;371:660-667.

11. Colombel JF, Rutgeerts P, Reinisch W, et al. Early mucosal healing with infliximab is associated with improved long-term clinical outcomes in ulcerative colitis. Gastroenterology 2011;141:1194-1201.

12. Colombel JF, Reinisch W, Mantzaris GJ, et al. Randomised clinical trial: deep remission in biologic and immunomodulator naive patients with Crohn's disease. A SONIC post hoc analysis. Aliment Pharmacol Ther 2015;41:734746.

13. Park SH, Yang SK, Park SK, et al. Long-term prognosis of Crohn's disease and its temporal change between 1981 and 2012: a hospital-based cohort study from Korea. Inflamm Bowel Dis 2014;20:488-494.

14. Lee HS, Park SH, Yang SK, et al. Long-term prognosis of ulcerative colitis and its temporal change between 1977 and 2013: a hospital-based cohort study from Korea. J Crohns Colitis 2015;9:147-155.

15. Solberg IC, Vatn MH, Hoie O, et al. Clinical course in Crohn's disease: results of a Norwegian population-based ten-year follow-up study. Clin Gastroenterol Hepatol 2007;5:1430-1438. 
16. Khanna R, Bressler B, Levesque BG, et al. Early combined immunosuppression for the management of Crohn's disease (REACT): a cluster randomised controlled trial. Lancet 2015;386:1825-1834.

17. Oh EH, Oh K, Han M, et al. Early anti-TNF/immunomodulator therapy is associated with better long-term clinical outcomes in Asian patients with Crohn's disease with poor prognostic factors. PLoS One 2017;12:eo177479.

18. Kim JY, Park DI, Han DS, et al. Comparing the clinical outcomes of young-onset and adult-onset ulcerative colitis: a multi-center Korean Association for the Study for Intestinal Diseases study. Korean J Intern Med 2017;32:6978.

19. Park Y, Cheon JH, Park YL, et al. Development of a novel predictive model for the clinical course of Crohn's disease: results from the CONNECT study. Inflamm Bowel Dis 2017;23:1071-1079.

20. Jones J, Loftus EV Jr, Panaccione R, et al. Relationships between disease activity and serum and fecal biomarkers in patients with Crohn's disease. Clin Gastroenterol Hepatol 2008;6:1218-1224.

21. Froslie KF, Jahnsen J, Moum BA, Vatn MH; IBSEN Group. Mucosal healing in inflammatory bowel disease: results from a Norwegian population-based cohort. Gastroenterology 2007;133:412-422.

22. Shah SC, Colombel JF, Sands BE, Narula N. Mucosal healing is associated with improved long-term outcomes of patients with ulcerative colitis: a systematic review and meta-analysis. Clin Gastroenterol Hepatol 2016;14:12451255 .

23. Colombel JF, Rutgeerts PJ, Sandborn WJ, et al. Adalimumab induces deep remission in patients with Crohn's disease. Clin Gastroenterol Hepatol 2014;12:414-422.

24. Peyrin-Biroulet L, Sandborn W, Sands BE, et al. Selecting Therapeutic Targets in Inflammatory Bowel Disease (STRIDE): determining therapeutic goals for treat-totarget. Am J Gastroenterol 2015;110:1324-1338.

25. Kim ES, Chen M, Lee J, Lee CK, Kim YS. Diagnosis of inflammatory bowel disease in Asia: the results of a multinational web-based survey in the 2(nd) Asian Organization for Crohn's and Colitis (AOCC) meeting in Seoul. Intest Res 2016;14:224-230.

26. Bryant RV, Burger DC, Delo J, et al. Beyond endoscopic mucosal healing in UC: histological remission better predicts corticosteroid use and hospitalisation over 6 years of follow-up. Gut 2016;65:408-414.
27. Wei SC, Chang TA, Chao TH, et al. Management of Crohn's disease in Taiwan: consensus guideline of the Taiwan Society of Inflammatory Bowel Disease. Intest Res 2017;15:285-310.

28. Panes J, Jairath V, Levesque BG. Advances in use of endoscopy, radiology, and biomarkers to monitor inflammatory bowel diseases. Gastroenterology 2017;152:362-373.

29. Colombel JF, Panaccione R, Bossuyt P, et al. Effect of tight control management on Crohn's disease (CALM): a multicentre, randomised, controlled phase 3 trial. Lancet 2017.

30. Yu Q, Mao R, Lian L, et al. Surgical management of inflammatory bowel disease in China: a systematic review of two decades. Intest Res 2016;14:322-332.

31. Park JJ, Yang SK, Ye BD, et al. Second Korean guidelines for the management of Crohn's disease. Intest Res 2017;15:38-67.

32. De Cruz P, Kamm MA, Hamilton AL, et al. Crohn's disease management after intestinal resection: a randomised trial. Lancet 2015;385:1406-1417.

33. Wright EK, Kamm MA, De Cruz P, et al. Measurement of fecal calprotectin improves monitoring and detection of recurrence of Crohn's disease after surgery. Gastroenterology 2015;148:938-947.

34. Eun CS. Is the therapeutic drug monitoring of anti-TNF agents necessary in Korean inflammatory bowel disease patients? Gut Liver 2017;11:3-4.

35. Mitrev N, Vande Casteele N, Seow CH, et al. Review article: consensus statements on therapeutic drug monitoring of anti-tumour necrosis factor therapy in inflammatory bowel diseases. Aliment Pharmacol Ther 2017;46:10371053 .

36. Papamichael K, Chachu KA, Vajravelu RK, et al. Improved long-term outcomes of patients with inflammatory bowel disease receiving proactive compared with reactive monitoring of serum concentrations of infliximab. Clin Gastroenterol Hepatol 2017;15:1580-1588.

37. Roblin X, Rinaudo M, Del Tedesco E, et al. Development of an algorithm incorporating pharmacokinetics of adalimumab in inflammatory bowel diseases. Am J Gastroenterol 2014;109:1250-1256.

38. Wu KC, Ran ZH, Gao X, et al. Adalimumab induction and maintenance therapy achieve clinical remission and response in Chinese patients with Crohn's disease. Intest Res 2016;14:152-163.

39. Williet N, Boschetti G, Fovet M, et al. Association between low trough levels of vedolizumab during induction ther- 
apy for inflammatory bowel diseases and need for additional doses within 6 months. Clin Gastroenterol Hepatol 2017;15:1750-1757.

40. Kaplan GG. The global burden of IBD: from 2015 to 2025 . Nat Rev Gastroenterol Hepatol 2015;12:720-727.

41. Lee JW, Choi CH, Park JH, et al. Clinical features of active tuberculosis that developed during anti-tumor necrosis factor therapy in patients with inflammatory bowel disease. Intest Res 2016;14:146-151.

42. Lee KM, Kim YS, Seo GS, Kim TO, Yang SK; IBD Study Group of the Korean Association for the Study of Intestinal Diseases. Use of thiopurines in inflammatory bowel disease: a consensus statement by the Korean Association for the Study of Intestinal Diseases (KASID). Intest Res
2015;13:193-207.

43. Torres J, Boyapati RK, Kennedy NA, Louis E, Colombel JF, Satsangi J. Systematic review of effects of withdrawal of immunomodulators or biologic agents from patients with inflammatory bowel disease. Gastroenterology 2015;149:1716-1730.

44. Louis E, Mary JY, Vernier-Massouille G, et al. Maintenance of remission among patients with Crohn's disease on antimetabolite therapy after infliximab therapy is stopped. Gastroenterology 2012;142:63-70.

45. Pariente B, Laharie D. Review article: why, when and how to de-escalate therapy in inflammatory bowel diseases. Aliment Pharmacol Ther 2014;40:338-353. 